\title{
Cosmological Constraints from 21cm Surveys After Reionization
}

\author{
Eli Visbal, ${ }^{1,2, \text { * }}$ Abraham Loeb, ${ }^{2}$ and Stuart Wyithe ${ }^{3}$ \\ ${ }^{1}$ Jefferson Laboratory of Physics, Harvard University, Cambridge, MA 02138 \\ ${ }^{2}$ Harvard-Smithsonian CfA, 60 Garden Street, Cambridge, MA 02138 \\ ${ }^{3}$ School of Physics, University of Melbourne, Parkville, Victoria, Australia
}

(Dated: November 1, 2018)

\begin{abstract}
$21 \mathrm{~cm}$ emission from residual neutral hydrogen after the epoch of reionization can be used to trace the cosmological power spectrum of density fluctuations. Using a Fisher matrix formulation, we provide a detailed forecast of the constraints on cosmological parameters that are achievable with this probe. We consider two designs: a scaled-up version of the MWA observatory as well as a Fast Fourier Transform Telescope. We find that $21 \mathrm{~cm}$ observations dedicated to post-reionization redshifts may yield significantly better constraints than next generation Cosmic Microwave Background (CMB) experiments. We find the constraints on $\Omega_{\Lambda}, \Omega_{\mathrm{m}} h^{2}$, and $\Omega_{\nu} h^{2}$ to be the strongest, each improved by at least an order of magnitude over the Planck CMB satellite alone for both designs. Our results do not depend as strongly on uncertainties in the astrophysics associated with the ionization of hydrogen as similar $21 \mathrm{~cm}$ surveys during the epoch of reionization. However, we find that modulation of the $21 \mathrm{~cm}$ power spectrum from the ionizing background could potentially degrade constraints on the spectral index of the primordial power spectrum and its running by more than an order of magnitude. Our results also depend strongly on the maximum wavenumber of the power spectrum which can be used due to non-linearities.
\end{abstract}

PACS numbers:

\section{INTRODUCTION}

Recently, there has been much interest in the feasibility of mapping the three-dimensional distribution of cosmic hydrogen through its spin-flip transition at a resonant rest frame wavelength of $21 \mathrm{~cm} \mathrm{[1,} \mathrm{2].} \mathrm{Several} \mathrm{first}$ generation experiments are being constructed to probe the epoch of reionization (MWA [3], LOFAR [4], PAPER [5], 21CMA [6]) and more ambitious designs are being planned (SKA [7]).

One driver for mapping hydrogen through $21 \mathrm{~cm}$ emission is to measure cosmological parameters from the underlying cosmic power spectrum. During the epoch of reionization (EoR), the $21 \mathrm{~cm}$ power spectrum is shaped mainly by the structure of ionized regions. Even without precise knowledge of the ionization power spectrum it is possible to isolate the cosmological power spectrum by exploiting anisotropies in redshift space due to peculiar velocities [8, 9].

Recent work [10, 11] has shown that the $21 \mathrm{~cm}$ power spectrum accessible during the EoR has the potential to put tight constraints on cosmological parameters; however, these constraints depend on model-dependent uncertainties, the most important of which involves the ionization power spectrum.

In this paper, we explore in detail the constraints achievable from mapping the residual cosmic hydrogen after reionization [12, 13, 14, 15, 16, 17]. The hydrogen resides in pockets of dense galactic regions which are selfshielded from the UV background, also known as damped

*evisbal@fas.harvard.edu
Lyman- $\alpha$ absorbers (DLAs) in quasar spectra [18]. In difference from traditional galaxy redshift surveys, $21 \mathrm{~cm}$ surveys do not need to resolve individual galaxies but rather are able to monitor the smooth variation in their cumulative $21 \mathrm{~cm}$ emission owing to their clustering on large scales [15].

Measuring the $21 \mathrm{~cm}$ power spectrum after reionization offers several key advantages as compared to the EoR. First, the near uniformity of the UV radiation field guarantees that the $21 \mathrm{~cm}$ power spectrum would reliably trace the underlying matter power spectrum. However, the UV background could introduce a scale dependent modulation to the $21 \mathrm{~cm}$ power spectrum as large as one percent [17]. This modulation could introduce degeneracies that would significantly weaken constraints on the spectral index of the primordial power spectrum and its running. Another advantage of probing low redshifts is that the brightness temperature of the galactic synchrotron emission scales as $(1+z)^{2.6}$. However, this advantage is offset by the fact that the mass weighted neutral fraction of hydrogen is only a few percent at redshifts $z \lesssim 6[19]$.

In this paper we use the Fisher matrix formalism to quantify how effectively futuristic surveys dedicated to post-reionization redshifts $(z \lesssim 6)$ can constrain cosmology. We consider both a survey with ten times the number of cross dipoles of the MWA [3] $(5000 \times 16=$ $8 \times 10^{4}$ ) but operating at higher frequencies, which we term MWA5000, and a Fast Fourier Transform Telescope (FFTT) with $10^{6}$ dipoles over a square kilometer area 20]. We also show results for the combination of these surveys and a next generation Cosmic Microwave Background (CMB) experiment (Planck).

This paper is structured as follows. In $\S \mathrm{II}$ we discuss the detectability of the $21 \mathrm{~cm}$ signal after reionization and 
in $\S I I I$ we discuss its power spectrum. In $\S I V$ we consider the details of our Fisher matrix calculation and in $\S \mathrm{V}$ we present its results. Finally, we discuss and summarize our conclusions in $\S \mathrm{VI}$ and $\S \mathrm{VII}$.

\section{DETECTABILITY OF THE 21CM SIGNAL AFTER REIONIZATION}

Reionization starts with ionized (HII) regions around galaxies which grow and eventually overlap. This overlap of HII regions characterizes the end of the EoR. It was once thought that the $21 \mathrm{~cm}$ signal would disappear after this transition because there is little neutral hydrogen left. Recent work has shown that this is not the case [12, 15, 17, 21, 22]. The detectability of the signal may not decline substantially following the end of the EoR because the Galactic synchrotron foreground is weaker at higher frequencies (lower redshifts of the $21 \mathrm{~cm}$ emission).

After reionization most of the remaining neutral hydrogen is expected to reside in DLAs. Observations have shown that out to $z \approx 4$ the cosmological density parameter of $\mathrm{HI}$ is $\Omega_{\mathrm{HI}} \approx 10^{-3}$ [19]. This corresponds to a mass-averaged neutral fraction of a few percent. It does not contribute significantly to the Ly $\alpha$ forest, which is mostly shaped by the much smaller volume-averaged neutral fraction. A $21 \mathrm{~cm}$ survey like the ones we discuss are sensitive to all neutral hydrogen within the survey volume because no galaxies are individually identified and thus there is no minimum threshold for the detection of individual galaxies.

Even though the majority of the neutral hydrogen resides in self shielded clumps, self absorption is not expected to significantly reduce the $21 \mathrm{~cm}$ signal. This is supported by $21 \mathrm{~cm}$ absorption studies of DLAs over the redshift interval $0<z \lesssim 3.4$, which exhibit an optical depth to $21 \mathrm{~cm}$ absorption of the radio flux from a background quasar of less than a few percent 23, 24]. These observations are supported by theoretical calculations of the $21 \mathrm{~cm}$ optical depth of neutral gas in high redshift minihalos [25]. We also note that the spin temperature in DLAs is much higher than the CMB temperature and thus the $21 \mathrm{~cm}$ signal is independent of the kinetic temperature of the gas [23].

At $z \approx 4$ the $21 \mathrm{~cm}$ brightness temperature contrast with the CMB will be roughly $0.5 \mathrm{mK}$. On scales of 10 comoving Mpc, the rms amplitude of density fluctuations is roughly $\sigma \approx 0.2$, so we expect $21 \mathrm{~cm}$ fluctuations at a level of $\approx 0.1 \mathrm{mK}$. This is only an order of magnitude or so less than the largest fluctuations expected during the EoR [26]. These fluctuations combined with the lower brightness temperature of galactic synchrotron emission, which scales as $(1+z)^{2.6}$, should provide a detectable signal. Previous work (e.g. Fig. 10 in Ref.[22]) shows that the signal to noise ratio of the $21 \mathrm{~cm}$ signal after reionization may be similar or even higher than that during the EoR. However, it is important to note that it will be necessary to build special instruments which are optimized to these redshifts to obtain the type of results discussed in this paper.

\section{21CM POWER SPECTRUM}

Next, we describe the $21 \mathrm{~cm}$ power spectrum after the epoch of reionization. A complete discussion of the relevant physics can be found in Refs. 11, 12, 15, 17]. The difference between the average brightness temperature of $21 \mathrm{~cm}$ emission at redshift $z$ and the CMB temperature is described by

$$
\bar{T}_{\mathrm{b}} \approx 26 \bar{x}_{\mathrm{H}}\left(\frac{\bar{T}_{\mathrm{s}}-T_{\mathrm{CMB}}}{\bar{T}_{\mathrm{s}}} \frac{\Omega_{\mathrm{b}} h^{2}}{0.022}\right)\left(\frac{0.15}{\Omega_{\mathrm{m}} h^{2}} \frac{1+z}{10}\right)^{1 / 2} \mathrm{mK},
$$

where $\bar{x}_{\mathrm{H}}$ is the global neutral hydrogen fraction and $T_{\mathrm{s}}$ is the HI spin temperature. In Fourier space, the power spectrum of $21 \mathrm{~cm}$ brightness fluctuations is defined by $(2 \pi)^{3} \delta^{3}\left(\mathbf{k}-\mathbf{k}^{\prime}\right) P_{21}(\mathbf{k}) \equiv\left\langle\Delta T_{\mathrm{b}}(\mathbf{k})^{*} \Delta T_{\mathrm{b}}\left(\mathbf{k}^{\prime}\right)\right\rangle$, where $\mathbf{k}$ is the wave-vector of a given Fourier mode and $\Delta T_{\mathrm{b}}(\mathbf{k})$ is the brightness temperature fluctuation in Fourier space.

Before giving its exact form, we stress that the most important feature of $P_{21}(\mathbf{k})$ after reionization is that it essentially traces the cosmological matter power spectrum, $P(k)$. Extracting the cosmological power spectrum will make it possible to place tight constraints on many cosmological parameters. During the EoR the situation is more complicated. $P_{21}(\mathbf{k})$ contains additional terms which depend on the ionization power spectrum and the cross power-spectrum between the ionization and matter distributions.

Following the derivation of Ref. [17] we find that after reionization

$$
P_{21}(\mathbf{k})=\tilde{T}_{\mathrm{b}}^{2} \bar{x}_{\mathrm{HI}}^{2} P(k)\left[B(\mathbf{k})+f \mu^{2}\right]^{2} .
$$

Here $\tilde{T}_{\mathrm{b}}=\bar{T}_{\mathrm{b}} /\left(\bar{x}_{\mathrm{HI}}\left(\bar{T}_{\mathrm{s}}-T_{\mathrm{CMB}}\right) / T_{\mathrm{S}}\right), B(\mathbf{k})$ is the average scale dependent, $\bar{x}_{\mathrm{HI}}$ mass-weighted bias for the DLA host galaxies, and $\mu=\cos \theta$ where $\theta$ is the angle between the line of sight and the wave-vector $\mathbf{k}[17,21]$. The factor $\left[B(\mathbf{k})+f \mu^{2}\right]^{2}$ arises due to redshift space anisotropies from line of sight peculiar velocities [8, 27, 28]. Here the growth index is defined as $f=d \ln D / d \ln a$, where $D(z)$ is the growth factor of density perturbations and $a$ is the cosmic scale factor. The bias factor arises because the residual neutral hydrogen is located in host galaxies which are biased with respect to the underlying dark matter distribution. This bias and the neutral hydrogen fraction are the only quantities in the above equation which do not depend solely on fundamental physics.

While the near uniformity of the ionizing UV background causes $P_{21}(\mathbf{k})$ to trace the cosmological power spectrum, fluctuations introduce a modulation that could modify the power spectrum by less than one percent [17]. This modulation of the power spectrum adds a scale dependent correction factor to the host galaxy bias. The average bias $B(\mathbf{k})$ is equal to the mean halo bias $\bar{b}$, multiplied by a factor $(1-K(k))$. In appendix $\mathrm{A}$, we derive 
an expression for $K(k)$ for the case where the mean-freepath of ionizing photons is independent of position. If the effect of the ionizing background is near the maximum which could be expected it may be necessary to model it in order to estimate cosmological parameters reliably. In $\S \mathrm{V}$, we show how cosmological constraints would likely be affected by degeneracies with nuisance parameters introduced by a scale dependent bias model.

The bias, $b$, for halos of a particular mass $M$ can be derived using the Press-Schechter formalism modified to include non-spherical collapse [29]

$$
\begin{aligned}
& b(M, z)=1+ \\
& \quad \frac{1}{\delta_{\mathrm{c}}}\left[\nu^{\prime 2}+b \nu^{\prime 2(1-c)}-\frac{\nu^{\prime 2 c} / \sqrt{a}}{\nu^{\prime 2 c}+b(1-c)(1-c / 2)}\right],
\end{aligned}
$$

where $\nu^{\prime 2}=a \delta_{\mathrm{c}}^{2} / \sigma^{2}(M, z), a=0.707, b=0.5$, and $c=$ 0.6 . Here $\sigma^{2}(M, z)$ is the variance of the density field smoothed on a mass scale $\mathrm{M}$ and $\delta_{\mathrm{c}}$ is the linear overdensity threshold for collapse at redshift $z$.

Assuming that the neutral gas to halo mass ratio is independent of mass, we can derive the mean bias at a particular redshift, $\bar{b}$, as the weighted average

$$
\bar{b}=\frac{\int_{M_{\min }}^{\infty} \frac{d n}{d M} b(M, z) M d M}{\int_{M_{\min }}^{\infty} \frac{d n}{d M} M d M},
$$

where $d n / d M$ is the Sheth-Tormen mass function of dark matter halos [29]. The minimum halo mass $M_{\min }$ defines the threshold for assembling heated gas out of the photo-ionized intergalactic medium, corresponding to a minimum virial temperature $T_{\mathrm{vir}} \approx 10^{5} \mathrm{~K}[30,31,32,33$, [34, 35]. The appropriate value of $M_{\text {min }}$ will depend on the mass of galaxies which host DLAs, however we find our results insensitive to the exact choice of $M_{\min }$ (see Fig. (4).

A constant virial temperature leads to the redshift dependence $M_{\min }=5.3 \times 10^{9}((1+z) / 4.5)^{-1.5} M_{\odot}$. Based on observations of DLAs [19], we assume a fiducial value of neutral hydrogen density to be $x_{\mathrm{HI} 1}=0.02$.

Previous work has suggested that at high redshifts the galaxy power spectrum may be affected by reionization [36, 37]. In [36] it is shown that fluctuations in the redshift of reionization in different locations leads to variation in the minimum mass of galaxies which can be assembled. This creates a scale dependent modulation of the power spectrum. Recent observations find that at $z \approx$ 3 galaxies with virial velocities $<50-70 \mathrm{~km} / \mathrm{s}$ contribute little to DLAs and that most DLAs are hosted within halos having viral velocities between $V=100-150 \mathrm{~km} / \mathrm{s}$ [38]. Since DLAs and thus most of the remaining neutral hydrogen after reionization is not found in the smallest galaxies we do not expect changes in the minimum mass of galaxies which are assembled to have an important effect on the $21 \mathrm{~cm}$ power spectrum.

In [37], the effects of reionization on the galaxy power spectrum for more massive galaxies are explored. Reionization is found to change the power spectrum, but this was due to reionization modifying the average stellar age of galaxies and hence the mass to light ratio. We do not expect this to significantly effect the neutral hydrogen hosted in galaxies. Additionally the modulation of the power spectrum was found to be approximately scale independent, which would not be degenerate with cosmological parameters discussed in this work.

\section{A. Non-Linear Effects on Baryon Acoustic Oscillations}

It has been recently shown that non-linear effects may change the baryon acoustic oscillations (BAO) signature at larger scales than those previously considered [39]. The erasure of $\mathrm{BAO}$ information due to non-linear effects can be written as

$$
\begin{aligned}
P_{\mathrm{b}, \mathrm{nl}}(k, \mu)= & P_{\mathrm{b}, \operatorname{lin}}(k) \times \\
& \quad \exp \left[-\frac{k^{2}}{2}\left(\left(1-\mu^{2}\right) \Sigma_{\perp}^{2}+\mu^{2} \Sigma_{\|}^{2}\right)\right]
\end{aligned}
$$

where $P_{\mathrm{b}}$ is the part of the power spectrum which contains the wiggles from the BAO, $\Sigma_{\perp}=\Sigma_{0} D(z), \Sigma_{\|}=$ $\Sigma_{0}(1+f) D(z), \Sigma_{0}=11.6 h^{-1} \mathrm{Mpc}$, and $D(z)$ is the growth function normalized to $(1+z)^{-1}$ at high redshifts. In our Fisher matrix calculations $\left(P_{\mathrm{b}, \mathrm{nl}}(k)+P_{\text {smooth }}(k)\right)$ is used in place of $P(k)$ in Eq. 2, $P_{\mathrm{b}, \text { lin }}(k)=P(k)-$ $P_{\text {smooth }}(k)$, where $P_{\text {smooth }}(k)$ is a low order polynomial fit to $P(k)$ in $\log -\log$ space. This fit removes the wiggles from the BAO, such that $P_{\mathrm{b}}$ is just the part of the power spectrum containing the BAO wiggles. Our choice of $\Sigma_{0}$ corresponds to the value determined by simulations and scaled linearly with $\sigma_{8}$ to fit our fiducial cosmology. The scales implied by $\Sigma_{\perp}$ and $\Sigma_{\|}$are held fixed in observable units such that the exponential suppression can be taken outside of the derivatives in the Fisher matrix formalism described below. This ensures that the BAO erasure does not in any way improve the forecasted errors on cosmological parameters.

We emphasize that the exponential suppression of the power spectrum in Eq. 5 is only applied to the the $\mathrm{BAO}$ wiggles after the smooth linear power has been subtracted off. The power spectrum used in the derivatives which enter the Fisher matrix described below is the sum of the wiggles suppressed by Eq. 5 and the unsuppressed smooth linear power spectrum. For the error on $P_{21}$ which enters the Fisher matrix we do not suppress either the wiggles or the smooth part of the power spectrum.

\section{FISHER MATRIX FORMULATION}

Given a set of parameters $\lambda_{i}$, the Fisher matrix formalism provides an estimation of the error for each of the parameters associated with some data set [40]. The 
$1 \sigma$ errors on parameters can be estimated as

$$
\Delta \lambda_{i}=\sqrt{F_{i i}^{-1}}
$$

where

$$
F_{i j}=\sum_{\text {pixels }} \frac{1}{\delta P_{12}^{2}}\left(\frac{\partial P_{21}}{\partial \lambda_{i}}\right)\left(\frac{\partial P_{21}}{\partial \lambda_{j}}\right)
$$

[9, 10], $P_{21}$ is the total $21 \mathrm{~cm}$ power spectrum, and $\delta P_{21}$ is the uncertainty on a measurement of the power spectrum. The relevant derivatives have been calculated using the transfer functions from CAMB [41].

We work in $\mathbf{u}$-space rather than $\mathbf{k}$-space as described in Refs. 9, 10] to simplify calculating Alcock-Paczynski effects. A radio interferometer directly measures visibilities,

$$
V(u, v, \nu)=\int d \hat{\mathbf{n}} \Delta T_{\mathrm{b}}(\hat{\mathbf{n}}, \nu) A_{\nu}(\hat{\mathbf{n}}) e^{2 \pi i(u, v) \cdot \hat{\mathbf{n}}},
$$

where $V$ is the visibility for a pair of antennae and $A_{\nu}$ is the contribution to the primary beam in the $\hat{\mathbf{n}}$ direction. Here we have used the flat sky approximation. This is appropriate even in the case of the FFTT, which images the entire hemisphere, because essentially all of the cosmological information in our surveys is found on small angular scales. The vector $\mathbf{u}_{\perp}=(u, v)$ corresponds to the number of wavelengths between the pair of antennae. Performing the Fourier transform $I(\mathbf{u})=\int d \nu V(u, v, \nu) \exp (2 \pi i \nu \eta)$, we obtain a signal in terms of $\mathbf{u}=u \hat{\mathbf{i}}+v \hat{\mathbf{j}}+\eta \hat{\mathbf{k}}$, where $\eta$ has units of time and $\hat{\mathbf{k}}$ is the unit vector along the line of sight. Note that there is a one to one correspondence between $\mathbf{k}$ and $\mathbf{u}$ given by $2 \pi \mathbf{u}_{\perp} / d_{A}=\mathbf{k}_{\perp}$ perpendicular to the line of sight and $2 \pi \mathbf{u}_{\|} / \tilde{y}=\mathbf{k}_{\|}$along the line of sight, where $d_{A}$ is the angular diameter distance to the observation and $\tilde{y}$ is the ratio of comoving distance to frequency interval. With the Fourier conventions above we have $P_{21}(\mathbf{k})=P_{21}(\mathbf{u}) d_{A}^{2} \tilde{y}$. Working in $\mathbf{u}$-space simplifies our calculation because $P_{21}(\mathbf{u})$ is measurable without cosmological assumptions and thus Eq. (7) can be applied directly. If we were to work in $\mathbf{k}$ space, the Alcock-Paczynski effect would distort $P_{21}(\mathbf{k})$ from Eq. (2) when we take derivatives with respect to cosmological parameters.

Because we are considering discrete sources, the total $21 \mathrm{~cm}$ power spectrum will be the sum of Eq. (2) and a shot noise term $P_{\text {shot }}=P_{21}(k) /\left(\bar{b}^{2} P(k) n_{\text {DLA }}\right)$, where $n_{\text {DLA }}$ is the effective number density of the galaxies which host DLAs, $P_{21}(k)$ is $P_{21}(\mathbf{k})$ along the line of sight, and $\bar{b}^{2} P(k)$ is the power spectrum of DLA hosting galaxies along the line of sight.

Any constant $|\mathbf{k}|$ and $\theta$ define an annulus of constant $P_{21}(\mathbf{u})$. The Fisher matrix is calculated from Eq. (7) by summing the contribution from annuli which fill all of $\mathbf{k}$-space accessible to an observation. We divide a $21 \mathrm{~cm}$ survey into redshift bins small enough for the redshift evolution of $P(k)$ across a bin to be negligible. A separate Fisher matrix is calculated for each redshift bin and then summed into a Fisher matrix which reflects the information about the entire survey.

To calculate the error in Eq. (7) we follow the work of Refs. 9, 42]. The error on $P_{21}$ for a particular $|\mathbf{k}|$ and $\theta$ is

$$
\delta P_{21}=\frac{P_{21}+P_{\text {shot }}+P_{N}}{\sqrt{N_{\mathrm{c}} \times N_{\text {fields }} \times B_{\text {tot }} / B}},
$$

where $P_{N}$ is the noise power spectrum of an interferometer, $B$ is the bandwidth over which foregrounds can be removed, $B_{\text {tot }}$ is the total accessible bandwidth, $N_{\text {fields }}$ is the number of fields being imaged, and $N_{\mathrm{c}}$ is the number of independent cells in $\mathbf{k}$-space. The number of cells in an annulus of constant $P_{21}(\mathbf{u})$ is well approximated by

$$
N_{\mathrm{c}}=2 \pi k^{2} \sin \theta \Delta k \Delta \theta \frac{V}{(2 \pi)^{3}},
$$

where $V$ is the comoving volume observed by the experiment, $\Delta \theta$ and $\Delta k$ are the angular and $\mathbf{k}$-space widths of the annulus. We choose $\Delta k$ and $\Delta \theta$ such that $P_{21}(\mathbf{u})$ is essentially constant. The noise of an interferometer is given by

$$
P_{\mathrm{N}}\left(u_{\perp}\right)=\left(\frac{\lambda^{2} T_{\mathrm{sys}}}{A_{\mathrm{e}}}\right)^{2} \frac{1}{t_{0} n\left(u_{\perp}\right)}
$$

where $\lambda=21 \mathrm{~cm} \times(1+z)$ is the observed wavelength, $T_{\text {sys }}$ is the system temperature of the interferometer, $A_{\mathrm{e}}$ is the effective area, $t_{0}$ is the total observing time, and $n\left(u_{\perp}\right)$ is the number density of baselines. This error is identical to that applicable in studies of the EoR with the exception of the shot noise [9, 10].

The sensitivity of $21 \mathrm{~cm}$ measurements will be impacted by how effectively foregrounds such as the galactic synchrotron emission can be removed. Refs. [9, 43, 44] suggest that after fitting out a low order polynomial, residual foregrounds in the power spectrum will be negligible if the frequency band over which foregrounds are removed, $B$, is substantially smaller than the total bandpass available. This is true for wave-vectors greater than $k_{\min }=2 \pi /(\tilde{y} B)$. Recent work estimating errors on cosmological parameters with $21 \mathrm{~cm}$ surveys during the EoR has used this assumption in modeling foreground removal 9 , 10, 11]. It is encouraging that we find parameter constraints to depend relatively weakly on the exact value of the bandwidth over which foregrounds are removed (see Fig. 2). We note that while we expect these assumptions pertaining to foreground removal to be robust, the true ability to remove foregrounds may not be known until first generation $21 \mathrm{~cm}$ instruments are operational.

There are several factors which limit the scales accessible to $21 \mathrm{~cm}$ surveys for parameter determination. As discussed above, we assume that foregrounds can not be effectively removed from pixels with $k<k_{\min }$. There is also a minimum accessible value of $k \sin \theta$ imposed by the minimum baseline, $b_{\text {min }} \approx \sqrt{A_{\text {phys }}}$. We exclude information for $k$ values above the linear scale. We compare 


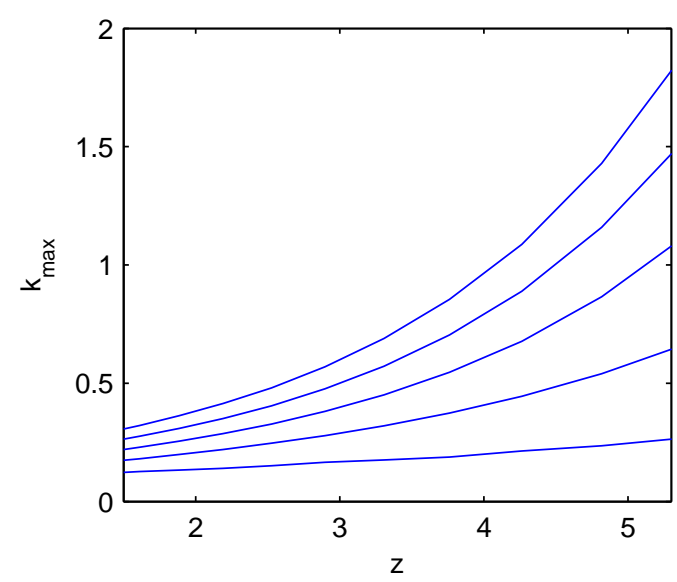

FIG. 1: Maximal wavenumber, $k_{\max }$, above which the non-linear power-spectrum deviates from the linear powerspectrum by more than $25 \%, 20 \%, 15 \%, 10 \%$ and $5 \%$ (top to bottom). Larger discrepancies produce higher values of $k_{\max }$.

the linear power spectrum for our fiducial cosmology to a non-linear power spectrum produced by HALOFIT in CAMB [41], and define our non-linear cutoff as the $k$ value where there is a $10 \%$ discrepancy.

The precision of the measurements discussed in this paper will require a theoretical prediction of the matter power spectrum which is accurate to better than one percent. The $k$ cutoff is meant to represent the scale to which future N-body simulations, such as those discussed in [45, 46], will be able to calculate the power spectrum to the required accuracy. Since the exact value of this scale is unknown we present the dependence of varying it on cosmological parameter constraints in Fig. 3 .

The value of this cutoff is fairly conservative when compared to the criterion of Ref. [47] which chooses $k_{\mathrm{nl}}=\pi / 2 R$ for a value of $R$ giving an $r m s$ density fluctuation amplitude $\sigma(R)=0.5$. The latter criterion has been used for $21 \mathrm{~cm}$ parameter constraints at higher redshifts than we explore [11]. The $k_{\max }$ values corresponding to different levels of discrepancy between the linear and non-linear power spectra are shown in Fig. 1.

We also include constraints when combined with future data from the Planck satellite [48]. The method used to calculate the corresponding Fisher matrix was adopted from Refs. [49, 50].

\section{A. Survey Characteristics}

We apply the Fisher matrix formulation to future surveys dedicated to low redshifts similar in design to MWA5000 and a Fast Fourier Transform Telescope (FFTT). We show results with surveys designed for a central redshift of 3.5 and 1.5 that each span a factor of 3 in $(1+z)$.

We model the MWA5000 observatory as an interferom- eter with 5000 tiles each containing 16 dipole antennae. For the survey centered at $z=3.5(1.5)$ we assume a constant core of antennae out to $40(22) \mathrm{m}$ and then a $r^{-2}$ distribution out to $580(278) \mathrm{m}$. This layout gives roughly the same baseline density distribution as MWA does at higher redshifts [9].

The angular resolution of the telescope is set by the baseline density distribution. The longest baseline corresponds to the highest value of $k_{\perp}$ that can be probed, $2 \pi \mathbf{u}_{\perp, \max } / d_{A}=\mathbf{k}_{\perp, \max }$. The above distribution of antennae contains baselines which are long enough to resolve $k_{\max }$ at all redshifts. We assume a frequency resolution of $0.01 \mathrm{MHz}$ for all surveys considered. These arrays will have better resolution than this, but this is sufficient to resolve the scales set by $k_{\max }$.

For the survey centered around $z=3.5$ the effective area of each tile $A_{\mathrm{e}} \approx N_{\mathrm{dip}} \lambda^{2} / 4$ and is limited by the physical area of the tile, $A_{\text {phys }}$ [51]. The values of $A_{\text {phys }}$ are chosen such that $A_{\mathrm{phys}} \approx A_{\mathrm{e}}$ at the central redshift. For the low-redshift case, we assume that dishes are used instead of antennae, resulting in $A_{\mathrm{e}} \approx A_{\text {phys }}$.

We model an FFTT observatory [20] as an interferometer with $10^{6}$ evenly spaced dipoles over a square kilometer that can all be correlated. The noise in each dipole is calculated separately with $A_{\mathrm{e}} \approx \lambda^{2} / 4$ limited by $A_{\text {phys }}=S^{2}$, where $S$ is the spacing of the dipoles on a square grid.

We have assumed that foregrounds can be removed on scales of up to $30[4.5 /(1+z)] \mathrm{MHz}$. This corresponds to the value that [15] cite as desirable to measure the neutrino signal. The exact choice of this bandwidth is relatively unimportant, as our results depend weakly on this value (see Fig. 2). We assume that 0.65 of the sky in one hemisphere is imaged for 2000 hours. This corresponds to roughly 16 fields of view. The partial coverage is due to foregrounds which can not be removed in the vicinity of the galactic plane. We also show results for a more conservative case using MWA5000 over three fields of view rather than 16 .

For all surveys we assume a redshift range corresponding to a factor of 3 in frequency and break each observation into redshift bins of width $\Delta z=0.1(1+z)$. The factor of 3 corresponds to the largest frequency bandwidth over which a low frequency dipole antenna has suitable sensitivity. In our lower redshift case we have assumed that dishes will be used. These could potentially have a larger bandwidth than the dipole antennae, but we conservatively use the same factor of 3 in frequency. We also assume that $T_{\text {sys }}=T_{\text {sky }}+T_{\text {inst }}$ where the sky temperature $T_{\text {sky }}=260[(1+z) / 9.5]^{2.55}[52]$, and that the instrumental temperature is $30 \mathrm{~K}$ in an optimistic case and $100 \mathrm{~K}$ in a pessimistic case, corresponding to what is reasonable with current technology and what might be expected by the time the hypothetical experiments we discuss will be built. 


\section{B. Cosmological Parameters}

Throughout this work we assume that the true background cosmology is that of a flat $\Lambda$ CDM Universe with density parameters $\Omega_{\Lambda}=0.7$ in dark energy, $\Omega_{\mathrm{m}} h^{2}=0.147$ in matter, $\Omega_{\mathrm{b}} h^{2}=0.023$ in baryons, and $\Omega_{\nu} h^{2}=0.00054$ in neutrinos, and with values of $A_{\mathrm{s}}^{2}=25.0 \times 10^{-10}, n_{\mathrm{s}}=0.95$, and $\alpha=0.0$ for the spectral index, amplitude, and running of the primordial power spectrum. The chosen value of $A_{\mathrm{s}}$ corresponds to an $r m s$ fluctuation amplitude on a $8 h^{-1} \mathrm{Mpc}$ scale of $\sigma_{8}=0.84$. We have assumed a neutrino hierarchy with one dominant species having a neutrino mass of $0.05 \mathrm{eV}$. We assume a fiducial value of $\tau=0.1$ for the optical depth to electron scattering during reionization, and a helium mass fraction from big bang nucleosynthesis of $Y_{\mathrm{He}}=0.24$. In a lower redshift example we also leave the dark energy equation of state as a free parameter with a fiducial value of $w=-1$.

\section{RESULTS}

We present the results of our Fisher matrix calculations in Tables [IVII] We consider several different sets of assumptions regarding the effects of the ionizing background, which as explained above, may introduce a weakly scale dependent bias. In principle this could produce degeneracies which would weaken constraints on cosmological parameters. First we present the case where the scale dependence of the bias is insignificant compared with the precision of the power spectrum measurements. Then we assume that the effect is large enough to require using a model with additional parameters to accurately fit the data. We also discuss a very pessimistic scenario where the form of the bias is completely unknown.

We calculate constraints for both optimistic and pessimistic antenna temperature, which are $30 \mathrm{~K}$ and $100 \mathrm{~K}$ respectively. We also show results for a cosmic variance limited version of each survey where the detector noise is assumed to make no contribution. This case represents the best conditions imaginable for constraining cosmological parameters given the finite volume of space that is observed. As it turns out, an FFTT is nearly cosmic variance limited at the redshifts of interest here. In this case, we only show results for the optimistic antenna temperature case.

We consider a flat $\Lambda$ CDM cosmology except for one case centered around $z=1.5$ where we leave $w$ as a free parameter.

\section{A. Constant Bias}

We begin with the case where the ionizing background does not introduce any significant modulation to the power spectrum. Thus, the bias, $B(\mathbf{k})=\bar{b}$ can be taken as a constant in each redshift bin. Note that the value of the bias is different in each redshift bin, by constant we are referring to its dependence on scale. This is the most optimistic model one could hope for. Here we assume that the shot noise, neutral fraction and the bias in each redshift bin are unknown. Each is marginalized over as a free parameter in the Fisher matrix. We use Eqs. (3) and (4) to determine the fiducial values of the bias. The shot noise is determined from the Press-Schechter mass function of dark matter halos modified to include non-spherical collapse. The fiducial value of the neutral fraction is set to $x_{\mathrm{HI}}=0.02$. The results are presented in Tables IIV.

\section{B. Scale Dependent Bias}

We consider the case where the fluctuations of the $21 \mathrm{~cm}$ power spectrum due to the ionizing background are large enough to require fitting additional nuisance parameters. We do not claim to derive the exact form of the modulation, instead we assume an idealized form and fit, and then see how cosmological constraints are degraded as more flexibility is introduced. As discussed above the scale dependent bias, $B(\mathbf{k})$, is the product of the average galaxy bias, $\bar{b}$ and $(1-K(\mathbf{k}))$. In [17], $K(\mathbf{k})$ is calculated for the case where all photons travel the same distance. Here we consider the more realistic case of a constant mean-free-path. In this case, as calculated in appendix $\mathrm{A}$, the modulation takes the form $K(\mathbf{k})=$ $K_{0} \arctan \left(k \lambda_{\mathrm{mfp}}\right) / \lambda_{\mathrm{mfp}} k$. We use the following approximation (given here in proper distance, but appearing in comoving distance in $K(\mathbf{k}))$ for the fiducial values of the mean-free-path: $\lambda_{\mathrm{mfp}}=85((1+z) / 4)^{-4} \mathrm{Mpc}$ [53]. As before, in our Fisher matrix calculations we marginalize over shot noise, neutral fraction and average bias in each redshift bin. Additionally, we marginalize over $K_{0}$ and $\lambda_{\text {mfp }}$ in each redshift bin. Our results are shown in Tables V.VI.

To see how constraints are affected as we increase the flexibility of the fit, we consider a case with small corrections to the form used above. Here we assume that the modulation is of the form

$$
K(\mathbf{k})=K_{0} \arctan \left(k \lambda_{\mathrm{mfp}}\right) / \lambda_{\mathrm{mfp}} k+c_{1} k+c_{2} k^{2}+c_{3} k^{3} \ldots
$$

where the $c_{n}$ 's are set to fiducial values of zero and marginalized over in each redshift bin. Aside from marginalizing over these new parameters, the Fisher matrix calculation is done the same as described above.

Table VII shows the results for FFTT for marginalizing over all of the $c_{n}$ 's up to degree n. This illustrates how parameter constraints will be degraded as there is more flexibility in the fit for $K(\mathbf{k})$. We find that most cosmological parameters are not degenerate with scale dependent changes to the bias. However, the spectral index of the primordial power spectrum and its running are degraded significantly. This is because a change in these parameters smoothly modifies the $21 \mathrm{~cm}$ power spectrum 
as a function of $\mathrm{k}$, which mimics changes due to scale dependent bias parameters. A change in other parameters alters features in the power spectrum that can be distinguished from the scale dependent bias. For example, one might expect $\Omega_{\nu} h^{2}$ to be strongly degenerate as well, since neutrinos change the power spectrum in a smooth way. However, with our choice of cosmological parameters, we hold $\Omega_{m} h^{2}$ constant when taking derivatives of $\Omega_{\nu} h^{2}$. This means that changing the energy density of neutrinos also changes the energy density of cold dark matter. The associated change in the cold dark matter component alters features in the power spectrum which break the degeneracy with the scale dependent bias.

Note that while the above fit gives a range of how much fitting the scale dependent bias could weaken cosmological constraints, future work will have to determine the exact form of the fit which should be used. This will require detailed numerical simulations.

We have also considered the extremely pessimistic case where the form of the scale dependent bias is completely unknown. It has been found that it may be possible to isolate the part of the power spectrum containing only cosmological information by exploiting the angular dependence of the power spectrum. We use the PESS ionization power spectrum model described in [10] to calculate Fisher matrices. This isolates the term in the $21 \mathrm{~cm}$ power spectrum which does not depend on the bias. We find that even FFTT cannot constrain cosmological effectively and does not improve constraints from Planck significantly. This is probably overly pessimistic since the changes to the bias from the ionizing background are expected to be small, but it shows the importance of understanding the form of the bias.

\section{Other Factors}

There are several other factors that affect the constraints attainable with post-reionization $21 \mathrm{~cm}$ surveys. We investigate how these factors change our constraints in the case of a constant bias. In Fig. 2, we have plotted the dependence of the $1 \sigma$ uncertainty on the bandwidth over which foregrounds are removed. Larger values for the bandwidth allow measurement of smaller $k$ values which are otherwise not accessible. Overall, the sensitivity to the bandwidth variation is relatively modest.

In Fig. 3, we show how the constraints vary for different choices of $k_{\max }$, the maximum wavenumber being used. We show how the parameter uncertainties change if we impose the cutoff at different levels of discrepancy between the non-linear and linear power spectra. As $k_{\max }$ is lowered errors on parameters are substantially increased. For a discrepancy of 0.1 and $0.05, k_{\max }$ ranges from $0.68-0.16 \mathrm{Mpc}^{-1}$ and $0.28-0.12 \mathrm{Mpc}^{-1}$ respectively, see Fig. 1. For a discrepancy of $0.01 k_{\max } \approx 0.06 \mathrm{Mpc}^{-1}$ for each redshift bin.

In Fig. 4, we show how the constraints depend on $M_{\text {min }}$, the minimum halo mass above which significant

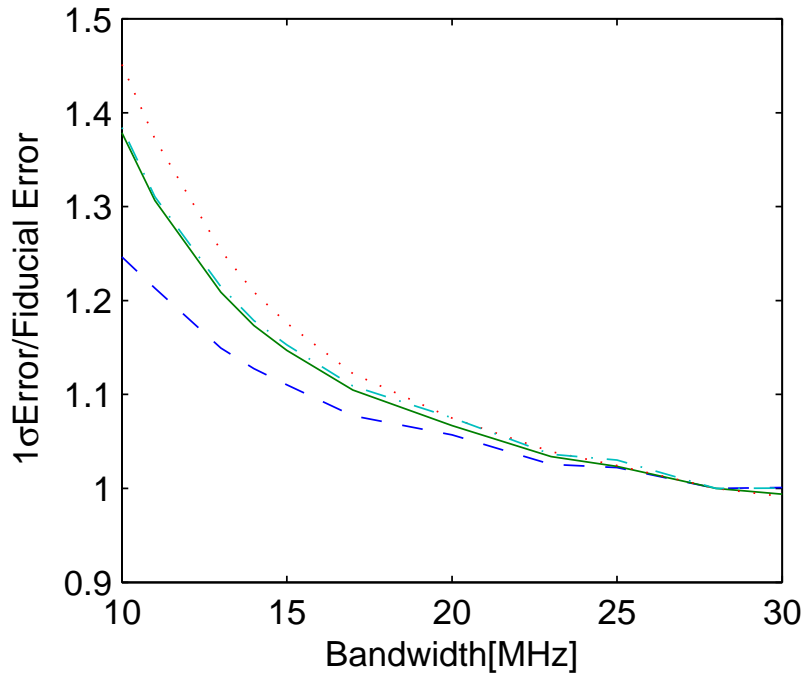

FIG. 2: Sensitivity of the relative $1 \sigma$ error bar to the bandwidth over which foregrounds can be removed for FFTT. Errors have been normalized such that they equal unity for the assumptions in Table I. The dashed line gives the error for $\Omega_{\Lambda}$, the dotted line for $\Omega_{\nu} h^{2}$, the solid line for $n_{\mathrm{s}}$ and the dot-dash line for $\alpha$.

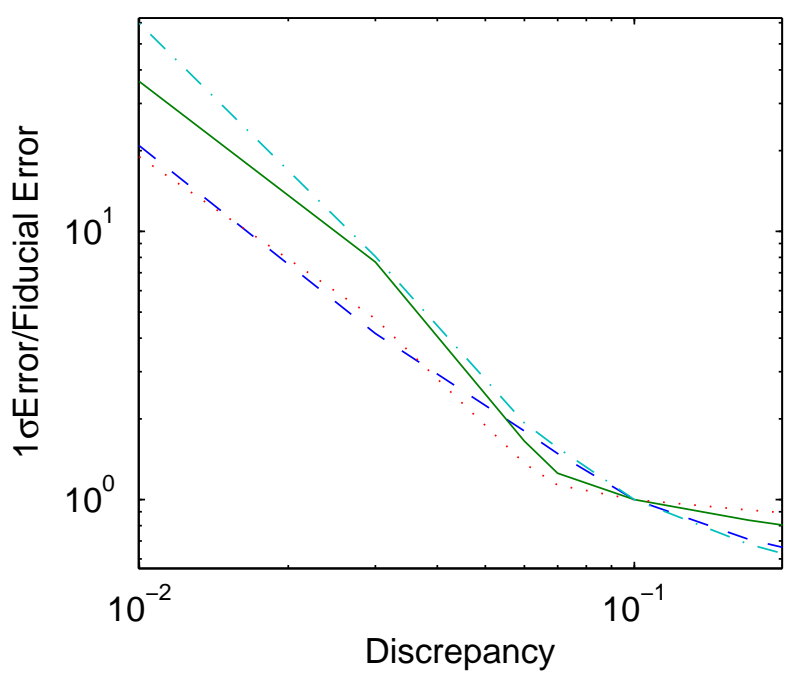

FIG. 3: Sensitivity of the relative $1 \sigma$ error bar to the maximal wavenumber $k_{\max }$ for FFTT. Errors have been normalized such that they equal one for the assumptions in Table 1 The dashed line gives the error for $\Omega_{\Lambda}$, the dotted line for $\Omega_{\nu} h^{2}$, the solid line for $n_{\mathrm{s}}$ and the dot-dash line for $\alpha$.

amounts of neutral hydrogen may assemble. A high value of $M_{\min }$ increases the shot noise and degrades the constraints. We find that the shot noise only affects the results significantly above $\sim 10^{12} M_{\odot}$, which is unrealistic since hydrogen is found in lower mass galaxies in the local Universe [54]. 
TABLE I: Constant Bias: $1 \sigma$ Errors on cosmological parameters with surveys centered at $z=3.5$. OPT refers to an antenna temperature of $30 \mathrm{~K}$, PESS 100K, and CVL to the cosmic variance limited case. OPT and PESS correspond to what may be possible by the time these experiments will be built and what is achievable today.

\begin{tabular}{|c|c|c|c|c|c|c|c|c|c|c|}
\hline & & $\Omega_{\Lambda}$ & $\Omega_{\mathrm{m}} h^{2}$ & $\Omega_{\mathrm{b}} h^{2}$ & $n_{\mathrm{S}}$ & $A_{\mathrm{S}}^{2} \times 10^{10}$ & $\alpha$ & $\Omega_{\nu} h^{2}$ & $\tau$ & $Y_{\mathrm{He}}$ \\
\hline Fiducial Values & & 0.7 & 0.147 & 0.023 & 0.95 & 25.0 & 0.0 & 0.00054 & 0.10 & 0.24 \\
\hline \multirow[t]{3}{*}{ MWA5k-Hemisphere } & PESS & 0.0041 & 0.0098 & 0.0024 & 0.03 & - & 0.012 & 0.0018 & - & - \\
\hline & OPT & 0.0024 & 0.0041 & 0.001 & 0.014 & - & 0.0068 & 0.00095 & - & - \\
\hline & $\mathrm{CVL}$ & 0.00059 & 0.00025 & 0.000074 & 0.003 & - & 0.0013 & 0.0003 & - & - \\
\hline \multirow[t]{3}{*}{ MWA5k-3 Fields } & PESS & 0.0095 & 0.023 & 0.0056 & 0.07 & $\overline{-}$ & 0.029 & 0.0042 & $\overline{-}$ & $\overline{-}$ \\
\hline & OPT & 0.0055 & 0.0096 & 0.0023 & 0.034 & - & 0.016 & 0.0022 & - & - \\
\hline & CVL & 0.0014 & 0.00059 & 0.00017 & 0.007 & - & 0.003 & 0.00071 & - & - \\
\hline FFTT & & 0.00076 & 0.00032 & 0.000087 & 0.0034 & $=$ & 0.0016 & 0.00032 & - & - \\
\hline Planck & & 0.038 & 0.0041 & 0.00024 & 0.0094 & 0.25 & 0.007 & 0.0039 & 0.0046 & 0.014 \\
\hline +MWA5k-Hemisphere & & 0.0023 & 0.00044 & 0.00013 & 0.0037 & 0.21 & 0.0028 & 0.00041 & 0.004 & 0.0057 \\
\hline +MWA5k-3 Fields & & 0.0053 & 0.00074 & 0.00017 & 0.0056 & 0.22 & 0.004 & 0.00077 & 0.0041 & 0.0082 \\
\hline +FFTT & & 0.00076 & 0.00022 & 0.000076 & 0.0027 & 0.2 & 0.0013 & 0.00026 & 0.004 & 0.0043 \\
\hline
\end{tabular}

${ }^{a}$ When not otherwise indicated the "OPT" value of $T_{\text {sys }}$ has been used.

TABLE II: Constant Bias: $1 \sigma$ Errors on cosmological parameters with surveys centered at $z=1.5$. OPT refers to an antenna temperature of $30 \mathrm{~K}$, PESS 100K, and CVL to the cosmic variance limited case. OPT and PESS correspond to what may be possible by the time these experiments will be built and what is achievable today.

\begin{tabular}{|c|c|c|c|c|c|c|c|c|c|c|}
\hline & & $\Omega_{\Lambda}$ & $\Omega_{\mathrm{m}} h^{2}$ & $\Omega_{\mathrm{b}} h^{2}$ & $n_{\mathrm{S}}$ & $A_{\mathrm{s}}^{2} \times 10^{10}$ & $\alpha$ & $\Omega_{\nu} h^{2}$ & $\tau$ & $Y_{\mathrm{He}}$ \\
\hline Fiducial Values & & 0.7 & 0.147 & 0.023 & 0.95 & 25.0 & 0.0 & 0.00054 & 0.10 & 0.24 \\
\hline \multirow[t]{3}{*}{ MWA5k-Hemisphere } & PESS & 0.0033 & 0.016 & 0.004 & 0.051 & - & 0.019 & 0.0027 & - & - \\
\hline & OPT & 0.0023 & 0.011 & 0.0027 & 0.036 & - & 0.014 & 0.002 & - & - \\
\hline & CVL & 0.0018 & 0.0085 & 0.0021 & 0.029 & - & 0.011 & 0.0016 & - & - \\
\hline \multirow{3}{*}{ MWA5k-3 Fields } & PESS & 0.0077 & 0.038 & 0.0093 & 0.12 & - & 0.044 & 0.0063 & - & - \\
\hline & OPT & 0.0054 & 0.026 & 0.0063 & 0.084 & - & 0.032 & 0.0046 & - & - \\
\hline & CVL & 0.0042 & 0.02 & 0.0048 & 0.068 & - & 0.026 & 0.0038 & - & - \\
\hline Planck & & 0.038 & 0.0041 & 0.00024 & 0.0094 & 0.25 & 0.007 & 0.0039 & 0.0046 & 0.014 \\
\hline +MWA5k-Hemisphere & & 0.0021 & 0.00042 & 0.00014 & 0.0042 & 0.22 & 0.0033 & 0.00041 & 0.0041 & 0.0065 \\
\hline +MWA5k-3 Fields & & 0.0047 & 0.00068 & 0.00018 & 0.0064 & 0.22 & 0.0048 & 0.00075 & 0.0041 & 0.0097 \\
\hline
\end{tabular}

${ }^{a}$ When not otherwise indicated the "OPT" value of $T_{\text {sys }}$ has been used.

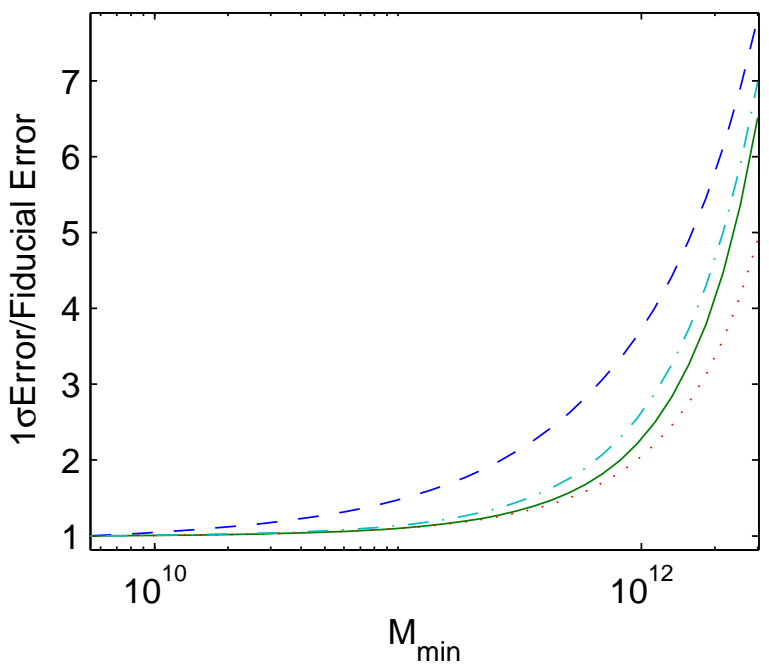

FIG. 4: Dependence of constraints on $M_{\min }$ (in units of solar mass) for FFTT. Errors have been normalized such that they equal one for the assumptions in table [1] The dashed line gives the error for $\Omega_{\Lambda}$, the dotted line for $\Omega_{\nu} h^{2}$, the solid line for $n_{\mathrm{s}}$ and the dot-dash line for $\alpha$.

\section{DISCUSSION}

The above results are very promising. We find that using the angular dependence of the power spectrum to isolate the term which only depends on cosmology cannot effectively constrain cosmological parameters. For this reason, it is crucial that the modulation of the $21 \mathrm{~cm}$ power spectrum from the ionizing background is modeled reliably.

Our results show approximately how constraints would be degraded if a fit were to be necessary. Table [VII] illustrates how more freedom in the fit affects cosmological constraints. We find that the only parameters which are strongly degenerate with the new fitting parameters are $n_{s}$ and $\alpha$, the spectral index and the running of the primordial power spectrum. The $1 \sigma$ errors on these parameters could increase by an order of magnitude. The other $1 \sigma$ cosmological parameter errors are increased by roughly $20 \%$ or less.

For comparison, the constraints from $21 \mathrm{~cm}$ surveys that focus on the EoR [10] vary considerably under different ionization power spectrum model assumptions. The robustness of our post-reionization constraints is mainly due to the fact that we do not have to consider the ionization power spectrum, only small scale dependent changes to the bias.

Our results also depend strongly on the maximum wavenumber of the power spectrum which can be used to reliably measure cosmological parameters, $k_{\max }$. It will be desirable for theoretical predictions of the matter power spectrum, presumably from N-body simulations, to be computed to a fraction of a percent for $k_{\max }$ values corresponding to a $5 \%$ descrepancy in the linear and non-linear power spectra shown in Fig. 1] If the matter 
TABLE III: Ratio of $1 \sigma$ Errors for surveys combined with Planck to Errors from Planck alone.

\begin{tabular}{|c|c|c|c|c|c|c|c|c|c|c|}
\hline & & $\Omega_{\Lambda}$ & $\Omega_{\mathrm{m}} h^{2}$ & $\Omega_{\mathrm{b}} h^{2}$ & $n_{\mathrm{S}}$ & $A_{\mathrm{S}}^{2}$ & $\alpha$ & $\Omega_{\nu} h^{2}$ & $\tau$ & $Y_{\mathrm{He}}$ \\
\hline MWA5k-Hemisphere & $z=3.5$ & 0.061 & 0.11 & 0.54 & 0.39 & 0.84 & 0.4 & 0.11 & 0.87 & 0.41 \\
\hline MWA5k-3 Fields & $z=3.5$ & 0.14 & 0.18 & 0.71 & 0.6 & 0.88 & 0.57 & 0.2 & 0.89 & 0.59 \\
\hline FFTT & $z=3.5$ & 0.02 & 0.054 & 0.32 & 0.29 & 0.8 & 0.19 & 0.067 & 0.87 & 0.31 \\
\hline MWA5k-Hemisphere & $z=1.5$ & 0.055 & 0.1 & 0.58 & 0.45 & 0.88 & 0.47 & 0.11 & 0.89 & 0.46 \\
\hline MWA5k-3 Fields & $z=1.5$ & 0.12 & 0.17 & 0.75 & 0.68 & 0.88 & 0.69 & 0.19 & 0.89 & 0.69 \\
\hline
\end{tabular}

"The "OPT" value of $T_{\text {sys }}(30 \mathrm{~K})$ and the constant bias model

have been used.

TABLE IV: Constant Bias: $1 \sigma$ Errors on cosmological parameters with surveys centered at $z=1.5$ with $w$ as a free parameter. OPT refers to an antenna temperature of 30K, PESS 100K, and CVL to the cosmic variance limited case. OPT and PESS correspond to what may be possible by the time these experiments will be built and what is achievable today.

\begin{tabular}{|c|c|c|c|c|c|c|c|c|c|c|c|}
\hline & & $\Omega_{\Lambda}$ & $\Omega_{\mathrm{m}} h^{2}$ & $\Omega_{\mathrm{b}} h^{2}$ & $n_{\mathrm{S}}$ & $A_{\mathrm{s}}^{2} \times 10^{10}$ & $\alpha$ & $\Omega_{\nu} h^{2}$ & $w$ & $\tau$ & $Y_{\mathrm{He}}$ \\
\hline Fiducial Values & & 0.7 & 0.147 & 0.023 & 0.95 & 25.0 & 0.0 & 0.00054 & -1.0 & 0.10 & 0.24 \\
\hline \multirow{3}{*}{ MWA5k-Hemisphere } & PESS & 0.0052 & 0.017 & 0.004 & 0.051 & - & 0.019 & 0.003 & 0.047 & - & - \\
\hline & OPT & 0.0047 & 0.012 & 0.0027 & 0.036 & - & 0.014 & 0.0022 & 0.039 & - & - \\
\hline & CVL & 0.0046 & 0.0088 & 0.0021 & 0.029 & - & 0.012 & 0.0018 & 0.036 & - & - \\
\hline \multirow{3}{*}{ MWA5k-3 Fields } & PESS & 0.012 & 0.039 & 0.0094 & 0.12 & - & 0.045 & 0.0069 & 0.11 & - & - \\
\hline & OPT & 0.011 & 0.027 & 0.0064 & 0.084 & - & 0.033 & 0.0051 & 0.092 & - & - \\
\hline & CVL & 0.011 & 0.02 & 0.0048 & 0.068 & - & 0.027 & 0.0043 & 0.083 & - & - \\
\hline Planck & & 0.096 & 0.0061 & 0.00024 & 0.0094 & 0.27 & 0.0071 & 0.0059 & 0.16 & 0.0051 & 0.015 \\
\hline +MWA5k-Hemisphere & & 0.0038 & 0.00056 & 0.00014 & 0.0042 & 0.22 & 0.0033 & 0.00049 & 0.023 & 0.0041 & 0.0065 \\
\hline +MWA5k-3 Fields & & 0.0082 & 0.00097 & 0.00018 & 0.0064 & 0.22 & 0.0048 & 0.0009 & 0.045 & 0.0041 & 0.0097 \\
\hline
\end{tabular}

${ }^{a}$ When not otherwise indicated the "OPT" value of $T_{\text {sys }}$ has been used.

TABLE V: Fit to Scale Dependent Bias: $1 \sigma$ Errors on cosmological parameters with surveys centered at $z=3.5$. OPT refers to an antenna temperature of $30 \mathrm{~K}$, PESS 100K, and CVL to the cosmic variance limited case. OPT and PESS correspond to what may be possible by the time these experiments will be built and what is achievable today.

\begin{tabular}{|c|c|c|c|c|c|c|c|c|c|c|}
\hline & & $\Omega_{\Lambda}$ & $\Omega_{\mathrm{m}} h^{2}$ & $\Omega_{\mathrm{b}} h^{2}$ & $n_{\mathrm{S}}$ & $A_{\mathrm{S}}^{2} \times 10^{10}$ & $\alpha$ & $\Omega_{\nu} h^{2}$ & $\tau$ & $Y_{\mathrm{He}}$ \\
\hline Fiducial Values & & 0.7 & 0.147 & 0.023 & 0.95 & 25.0 & 0.0 & 0.00054 & 0.10 & 0.24 \\
\hline \multirow{3}{*}{ MWA5k-Hemisphere } & PESS & 0.0043 & 0.011 & 0.0027 & 0.047 & - & 0.028 & 0.002 & - & $\overline{-}$ \\
\hline & OPT & 0.0025 & 0.0046 & 0.0011 & 0.028 & - & 0.017 & 0.001 & - & - \\
\hline & CVL & 0.00061 & 0.00026 & 0.000077 & 0.007 & - & 0.0031 & 0.00033 & - & - \\
\hline \multirow{3}{*}{ MWA5k-3 Fields } & PESS & 0.01 & 0.026 & 0.0062 & 0.11 & - & 0.066 & 0.0047 & - & - \\
\hline & OPT & 0.0058 & 0.011 & 0.0026 & 0.065 & - & 0.04 & 0.0024 & - & - \\
\hline & CVL & 0.0014 & 0.0006 & 0.00018 & 0.016 & - & 0.0072 & 0.00078 & - & - \\
\hline FFTT & & 0.00079 & 0.00033 & 0.000089 & 0.0085 & - & 0.0041 & 0.00035 & - & - \\
\hline Planck & & 0.038 & 0.0041 & 0.00024 & 0.0094 & 0.25 & 0.007 & 0.0039 & 0.0046 & 0.014 \\
\hline +MWA5k-Hemisphere & & 0.0024 & 0.00052 & 0.00013 & 0.0042 & 0.22 & 0.0032 & 0.00051 & 0.0041 & 0.007 \\
\hline +MWA5k-3 Fields & & 0.0055 & 0.00078 & 0.00017 & 0.0063 & 0.22 & 0.0048 & 0.00094 & 0.0041 & 0.01 \\
\hline +FFTT & & 0.00079 & 0.00025 & 0.000078 & 0.0034 & 0.21 & 0.0017 & 0.00029 & 0.004 & 0.0052 \\
\hline
\end{tabular}

${ }^{a}$ When not otherwise indicated the "OPT" value of $T_{\text {sys }}$ has been used.

TABLE VI: Fit to Scale Dependent Bias: $1 \sigma$ Errors on cosmological parameters with surveys centered at $z=1.5$. OPT refers to an antenna temperature of $30 \mathrm{~K}$, PESS 100K, and CVL to the cosmic variance limited case. OPT and PESS correspond to what may be possible by the time these experiments will be built and what is achievable today.

\begin{tabular}{|c|c|c|c|c|c|c|c|c|c|c|}
\hline & & $\Omega_{\Lambda}$ & $\Omega_{\mathrm{m}} h^{2}$ & $\Omega_{\mathrm{b}} h^{2}$ & $n_{\mathrm{S}}$ & $A_{\mathrm{s}}^{2} \times 10^{10}$ & $\alpha$ & $\Omega_{\nu} h^{2}$ & $\tau$ & $Y_{\mathrm{He}}$ \\
\hline Fiducial Values & & 0.7 & 0.147 & 0.023 & 0.95 & 25.0 & 0.0 & 0.00054 & 0.10 & 0.24 \\
\hline \multirow[t]{3}{*}{ MWA5k-Hemisphere } & PESS & 0.0034 & 0.019 & 0.0047 & 0.067 & - & 0.034 & 0.0032 & - & - \\
\hline & OPT & 0.0024 & 0.013 & 0.0031 & 0.048 & - & 0.025 & 0.0023 & - & - \\
\hline & CVL & 0.0019 & 0.0095 & 0.0023 & 0.039 & - & 0.02 & 0.0019 & - & - \\
\hline \multirow[t]{3}{*}{ MWA5k-3 Fields } & PESS & 0.008 & 0.045 & 0.011 & 0.16 & - & 0.08 & 0.0076 & $=$ & $=$ \\
\hline & OPT & 0.0056 & 0.03 & 0.0072 & 0.11 & - & 0.058 & 0.0054 & - & - \\
\hline & CVL & 0.0043 & 0.022 & 0.0053 & 0.09 & - & 0.046 & 0.0044 & - & - \\
\hline Planck & & 0.038 & 0.0041 & 0.00024 & 0.0094 & 0.25 & 0.007 & 0.0039 & 0.0046 & $\overline{0.014}$ \\
\hline +MWA5k-Hemisphere & & 0.0021 & 0.00049 & 0.00014 & 0.0045 & 0.22 & 0.0037 & 0.00053 & 0.0041 & 0.0073 \\
\hline +MWA5k-3 Fields & & 0.0048 & 0.00071 & 0.00018 & 0.0069 & 0.22 & 0.0052 & 0.00092 & 0.0042 & 0.011 \\
\hline
\end{tabular}

${ }^{a}$ When not otherwise indicated the "OPT" value of $T_{\text {sys }}$ has been used.

TABLE VII: Polynomial Corrections to Bias: $1 \sigma$ Errors on cosmological parameters for FFTT centered around $z=3.5$. Effects of fitting small polynomial corrections to the scale dependent bias. Here $\mathrm{n}$ is the degree of the polynomial marginalized over. The case with $\mathrm{n}=0$ corresponds to the value from table $\mathrm{V}$

\begin{tabular}{|c|c|c|c|c|c|c|c|}
\hline & $\Omega_{\Lambda}$ & $\Omega_{\mathrm{m}} h^{2}$ & $\Omega_{\mathrm{b}} h^{2}$ & $n_{\mathrm{S}}$ & $A_{\mathrm{s}}^{2} \times 10^{10}$ & $\alpha$ & $\Omega_{\nu} h^{2}$ \\
\hline Fiducial Values & 0.7 & 0.147 & 0.023 & 0.95 & 25.0 & 0.0 & 0.00054 \\
\hline constant bias & 0.00076 & 0.00032 & 0.000087 & 0.0034 & - & 0.0016 & 0.00032 \\
\hline $\mathrm{n}=0$ & 0.00079 & 0.00033 & 0.000089 & 0.0085 & - & 0.0041 & 0.00035 \\
\hline $\mathrm{n}=1$ & 0.00084 & 0.00033 & 0.00009 & 0.011 & - & 0.0071 & 0.00036 \\
\hline $\mathrm{n}=2$ & 0.00092 & 0.00035 & 0.000093 & 0.016 & - & 0.014 & 0.00036 \\
\hline $\mathrm{n}=3$ & 0.00093 & 0.00037 & 0.000097 & 0.017 & - & 0.015 & 0.00037 \\
\hline $\mathrm{n}=4$ & 0.00093 & 0.00038 & 0.0001 & 0.017 & - & 0.015 & 0.00037 \\
\hline
\end{tabular}


power spectrum can not be computed to this accuracy, the value of $k_{\max }$ may be the limiting factor for measuring cosmological parameters with experiments similar to those described in this paper.

The noise in the interferometers is lower at lower redshift due to the redshift scaling of the galactic synchrotron emission. This advantage is offset by the fact that at higher redshifts the neutral fraction is higher and the observed power spectrum stays linear up to a higher $k_{\max }$ value. The FFTT and the MWA5000 at $z=1.5$ are nearly cosmic variance limited. These experiments would be close to capturing all the information available from the power spectrum available to us on linear scales at the relevant redshifts. The MWA5000 with optimistic antenna temperature centered at $z=3.5$ gives errors roughly five times larger than the cosmic variance limited case.

Current cosmological information sets the upper limit

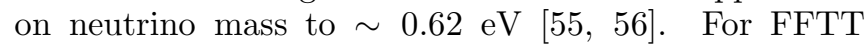
with a constant bias, we find a $1 \sigma$ error corresponding to $0.030 \mathrm{eV}$. As expected [15], this is clearly much better than existing constraints and is sufficiently small to probe the neutrino mass differences implied by neutrino oscillations.

It is also interesting to compare how well these surveys constrain cosmology with respect to other future probes. The FFTT survey will perform much better than the Planck satellite for almost all of the parameters under consideration. When combined with Planck, essentially all of the constraints are improved. In Table III we explicitly show how the constraint on each parameter from Planck would improve if combined with post-reionization $21 \mathrm{~cm}$ surveys. The most improved parameters are $\Omega_{\Lambda}$, $\Omega_{\mathrm{m}} h^{2}$, and $\Omega_{\nu} h^{2}$, which for FFTT are $2.0 \%, 5.4 \%$, and $6.7 \%$ of the errors from Planck alone.

Since most of the remaining neutral hydrogen after the EoR is found in DLAs hosted by galaxies, we expect our results to be similar to galaxy redshift surveys over similar redshift ranges. In [11] an all sky galaxy survey centered around $z=1$, termed G3, is considered. We find the cosmological constraints they calculate to be very similar to those in our MWA5000 case with a constant bias centered around $z=1.5$. They also consider a Lyman break survey centered around $z=3$, which they term G2. This survey contains roughly $1 \%$ of the volume included in our MWA5000-hemisphere and FFTT cases. Since the error on the power spectrum scales as the square root of the volume of a survey, this should cause parameter errors in G2 to be roughly an order of magnitude larger. We find that this falls roughly into the range we calculate above for the constant bias MWA5000 case centered around $z=3.5$. Note that if the degeneracies due to the UV background discussed above are present, galaxy surveys may do significantly better than $21 \mathrm{~cm}$ at measuring the $n_{s}$ and $\alpha$, the spectral index of the primordial power spectrum and its running.

In the case where we allow $w$ to vary as a free parameter we find the constraints on most parameters es- sentially unchanged. The main exception is $\Omega_{\Lambda}$, where we find that our constraints are increased by roughly a factor of two. The constraints achievable for $w$ are interesting, about $3.9 \%$ if the whole hemisphere is observed, and $9.2 \%$ with only three fields of view.

\section{CONCLUSIONS}

A $21 \mathrm{~cm}$ survey after reionization provides a promising probe of cosmology and fundamental physics [15]. We find the simple form of the $21 \mathrm{~cm}$ power spectrum after reionization provides less uncertainty in forecasting constraints than $21 \mathrm{~cm}$ during the EoR. However, we find that errors on estimates of the spectral index and the running of the primordial power spectrum could be increased by an order of magnitude if the ionizing background introduces scale dependent effects into the post reionization $21 \mathrm{~cm}$ power spectrum.

Aside from its greater simplicity, the measurement sensitivity after reionization is improved over EoR surveys due to lower foreground brightness temperature, a bias that is greater than unity, and a larger growth factor of density perturbations. These advantages are somewhat offset by the fact that there is less neutral hydrogen after reionization.

The constraints we derive are competitive with those from the EoR and for the FFTT are significantly better than next generation CMB experiments on their own. We find errors on $\Omega_{\Lambda}, \Omega_{\mathrm{m}} h^{2}$, and $\Omega_{\nu} h^{2}$ to be improved the most, each lowering the errors from Planck alone by at least an order of magnitude for both FFTT and the survey similar in design to MWA5000.

\section{APPENDIX: MODULATION OF 21CM PS FROM IONIZING BACKGROUND}

We derive the form of the modulation of the $21 \mathrm{~cm}$ power spectrum due to the ionizing background in the case of a constant mean-free-path. The only difference in the derivation presented in [17] is the form of the fluctuations of the ionizing flux $\delta_{J}(\mathbf{x})$. We convolve the realspace density field with a filter function to account for the effects of a finite mean-free-path and the inverse squared dependence of ionizing flux. The fluctuation in flux is given by

$$
\delta_{J}(\mathbf{x}) \propto \int d \mathbf{x}^{\prime} G\left(\mathbf{x}, \mathbf{x}^{\prime}\right) \delta\left(\mathbf{x}^{\prime}\right)
$$

We assume a mean-free-path of ionizing photons which is independent of position such that,

$$
G\left(\mathbf{x}, \mathbf{x}^{\prime}\right) \propto \frac{\exp \left(-\left|\mathbf{x}^{\prime}-\mathbf{x}\right| / \lambda_{\mathrm{mfp}}\right)}{\left|\mathbf{x}^{\prime}-\mathbf{x}\right|^{2}}
$$


where $\lambda_{\mathrm{mfp}}$ is the mean free path of ionizing photons. The convolution theorem gives

$$
\delta_{J}(\mathbf{k}) \propto g(\mathbf{k}) \delta_{k},
$$

where $g(\mathbf{k})$ and $\delta_{k}$ are the Fourier transforms of the filter function and the fluctuation in ionizing flux. We find that

$$
g(\mathbf{k}) \propto \arctan \left(k \lambda_{\operatorname{mfp}}\right) / k .
$$

From the derivation presented in [17], this implies that the power spectrum is given by Eqn. 2 with

$$
K(k)=K_{0} \arctan \left(k \lambda_{\mathrm{mfp}}\right) / \lambda_{\mathrm{mfp}} k,
$$

where $K_{0}$ is a constant of the order of 0.01 or smaller [17].

\section{ACKNOWLEDGMENTS}

This work was supported in part by a NASA LA grant and by Harvard University funds. We thank Matt McQuinn and Jonathan Pritchard for useful discussions.
[1] S. Furlanetto, S. P. Oh, and F. Briggs, Phys. Rept. 433, 181 (2006).

[2] R. Barkana and A. Loeb, Reports on Progress in Physics 70, 627 (2007).

[3] http://www.haystack.mit.edu/ast/arrays/mwa.

[4] http://www.lofar.org.

[5] http://www.astro.berkeley.edu/dbacker/EoR.

[6] http://web.phys.cmu.edu/ past.

[7] http://www.skatelescope.org.

[8] R. Barkana and A. Loeb, Astrophys. J. 624, L65 (2005).

[9] M. McQuinn, O. Zahn, M. Zaldarriaga, L. Hernquist, and S. R. Furlanetto, Astrophys. J. 653, 815 (2006).

[10] Y. Mao, M. Tegmark, M. McQuinn, M. Zaldarriaga, and O. Zahn, Phys. Rev. D 78, 023529 (2008).

[11] J. R. Pritchard and E. Pierpaoli, Phys. Rev. D 78, 065009 (2008).

[12] S. Wyithe and A. Loeb, Mon. Not. Roy. Astron. Soc. 383, 606 (2007).

[13] J. S. B. Wyithe, A. Loeb, and P. M. Geil, Mon. Not. Roy. Astron. Soc. 383, 1195 (2008).

[14] T.-C. Chang, U.-L. Pen, J. B. Peterson, and P. McDonald, Phys. Rev. Lett. 100, 091303 (2008).

[15] A. Loeb and J. S. B. Wyithe, Physical Review Letters 100, 161301 (2008)

[16] U.-L. Pen, L. Staveley-Smith, J. Peterson, and T.-C. Chang, ArXiv e-prints (2008), 0802.3239.

[17] S. Wyithe and A. Loeb, ArXiv e-prints (2008), 0808.2323.

[18] A. M. Wolfe, E. Gawiser, and J. X. Prochaska, Ann. Rev. Astron. Astrophys. 43, 861 (2005).

[19] J. X. Prochaska, S. Herbert-Fort, and A. M. Wolfe, Astrophys. J. 635, 123 (2005).

[20] M. Tegmark and M. Zaldarriaga, ArXiv e-prints 805 (2008), 0805.4414

[21] S. Wyithe, Mon. Not. Roy. Astron. Soc. 388, 1889 (2008).

[22] J. R. Pritchard and A. Loeb, Phys. Rev. D78, 103511 (2008).

[23] N. Kanekar and J. N. Chengalur, Astron. Astrophys. 399, 857 (2003).

[24] S. J. Curran, P. Tzanavaris, M. T. Murphy, J. K. Webb, and Y. M. Pihlstroem, Mon. Not. Roy. Astron. Soc. 381, L6 (2007).

[25] S. Furlanetto and A. Loeb, Astrophys. J. 579, 1 (2002).

[26] S. Wyithe and M. Morales, Mon. Not. Roy. Astron. Soc. 379, 1647 (2007).

[27] N. Kaiser, Mon. Not. Roy. Astron. Soc. 227, 1 (1987).
[28] S. Bharadwaj and S. S. Ali, Mon. Not. Roy. Astron. Soc. 352, 142 (2004).

[29] R. K. Sheth, H. J. Mo, and G. Tormen, Mon. Not. Roy. Astron. Soc. 323, 1 (2001).

[30] S. Wyithe and A. Loeb, Nature 441, 332 (2006).

[31] G. Efstathiou, Mon. Not. Roy. Astron. Soc. 256, 43P (1992).

[32] P. R. Shapiro, M. L. Giroux, and A. Babul, Astrophys. J. 427, 25 (1994).

[33] A. A. Thoul and D. H. Weinberg, Astrophys. J. 465, 608 (1996).

[34] L. Hui and N. Y. Gnedin, Mon. Not. Roy. Astron. Soc. 292, 27 (1997).

[35] A. Mesinger and M. Dijkstra, Mon. Not. Roy. Astron. Soc. 390, 1071 (2008).

[36] D. Babich and A. Loeb, Astrophys. J. 640, 1 (2006).

[37] S. Wyithe and A. Loeb, Mon. Not. Roy. Astron. Soc. 382, 921 (2007).

[38] L. A. Barnes and M. G. Haehnelt, Mon. Not. Roy. Astron. Soc. 397, 511 (2009), 0809.5056.

[39] H.-J. Seo and D. J. Eisenstein, Astrophys. J. 665, 14 (2007).

[40] M. Tegmark, A. N. Taylor, and A. F. Heavens, Astrophys. J. 480, 22 (1997).

[41] http://camb.info/.

[42] M. F. Morales, Astrophys. J. 619, 678 (2005).

[43] X.-M. Wang, M. Tegmark, M. Santos, and L. Knox, Astrophys. J. 650, 529 (2006).

[44] V. Jelić, S. Zaroubi, P. Labropoulos, R. M. Thomas, G. Bernardi, M. A. Brentjens, A. G. de Bruyn, B. Ciardi, G. Harker, L. V. E. Koopmans, et al., Mon. Not. Roy. Astron. Soc. 389, 1319 (2008).

[45] K. Heitmann, M. White, C. Wagner, S. Habib, and D. Higdon, ArXiv e-prints (2008), 0812.1052.

[46] K. Heitmann, D. Higdon, M. White, S. Habib, B. J. Williams, and C. Wagner, ArXiv e-prints (2009), 0902.0429 .

[47] H.-J. Seo and D. J. Eisenstein, Astrophys. J. 598, 720 (2003).

[48] http://sci.esa.int/planck/.

[49] G. Jungman, M. Kamionkowski, A. Kosowsky, and D. N. Spergel, Phys. Rev. D 54, 1332 (1996).

[50] M. Zaldarriaga and U. Seljak, Phys. Rev. D 55, 1830 (1997).

[51] J. D. Bowman, M. F. Morales, and J. N. Hewitt, Astro- 
phys. J. 638, 20 (2006).

[52] A. E. E. Rogers and J. D. Bowman (2008), 0806.2868.

[53] C. A. Faucher-Giguere, A. Lidz, L. Hernquist, and M. Zaldarriaga, Astrophys. J. 688, 85 (2008).

[54] J. I. Davies et al., Mon. Not. Roy. Astron. Soc. 328, 1151 (2001).
[55] A. Goobar, S. Hannestad, E. Mortsell, and H. Tu, JCAP 0606, 019 (2006).

[56] U. Seljak, A. Slosar, and P. McDonald, JCAP 0610, 014 (2006). 